\title{
Antecedentes urbanos, infraestructura de esparcimiento y recreación. Parques
}

\section{Lineales}

DOI: $10.20396 /$ labore.v13i0.8656735

Submetido 20 set. 2019.

Aceito 08 nov. 2019.

Publicado 03 der. 2019.

\author{
Mario Alberto Franco Muñoz \\ $<$ https://orcid.org/> \\ Universidad Autónoma de Aguascalientes / Aguascalientes [México]
}

\section{RESUMEN}

Hoy en día podemos encontrarnos con la grata sorpresa alrededor del mundo y en particular tanto en América latina, como en Europa y en nuestro país México, con el desarrollo de parques lineales, ejemplos de intervenciones de infraestructura urbana, en específico de esparcimiento y recreación, los cuales han tomado la escena de la nueva modalidad, en el diseño de los espacios públicos, en diversas ciudades, marcando el inicio y la pauta a seguir en estas prácticas del nuevo diseño urbano, en una búsqueda de revalorar y recuperar espacios naturales (lechos de ríos, barrancas, cuerpos de agua, etc.). De belleza intrínseca y de gran valor en cuanto a recursos naturales de flora y fauna, que a través de los últimos tiempos, por costumbre o inercia colectiva, se dejaban en un segundo término o marginados, como áreas o zonas territoriales de desecho. Consideradas como áreas con un bajo potencial, para el desarrollo de proyectos de esparcimiento y recreación o simplemente calificadas como áreas de desecho en las ciudades, las cuales no eran consideradas, como espacios naturales que intrínsecamente aportaban beneficios para el óptimo desarrollo de las ciudades, con el potencial que sus propias características ofrecen, si no por el contrario, catalogadas como zonas inconvenientes; permaneciendo en la mayoría de los casos abandonadas, sin atención por parte de las autoridades, convertidas en focos de contaminación y en áreas de inseguridad para las ciudades, sin llevar a cabo un intento consiente de la integración optima de estas, en los planes de desarrollo de nuestras ciudades en general. Es así, que se cuenta, como se mencionó anteriormente en América latina, Europa, así como en nuestro México, con varios ejemplos de intervenciones de infraestructura urbana de esparcimiento y recreación, en particular de parques urbanos lineales, que como nueva modalidad de espacio público se han desarrollado en diversas ciudades. Los cuales son fieles ejemplos, de cómo se pretende, de manera asertiva, aprovechar y dar un uso positivo a estas áreas naturales, ya sean los cauces de ríos, barrancas y cuerpos de agua (bordos, presas, lagos, etc.).

PALABRAS CLAVE

Parque lineal. Calidad de vida. Habitabilidad.

\section{Antecedentes urbanos, iniraestructura de esparcimiento y recreación. Parques Lineales}

\section{ABSTRACT}

Today we can find a pleasant surprise around the world and particularly in Latin America, Europe and Mexico, with the development of linear parks, examples of urban infrastructure interventions, specifically leisure and recreation, which have taken the scene of the new modality, in the design of public spaces, in various cities, marking the beginning and the pattern to follow in these practices of the new urban design, in a search to revalue and recover natural spaces (beds) of rivers, ravines, bodies of water, etc.). Of intrinsic beauty and great value in terms of natural resources of flora and fauna, which through recent times, due to collective habit or inertia, were left in a second term or marginalized, as areas or territorial waste areas. Considered as areas with low potential, for the development of leisure and recreation projects or simply classified as waste areas in cities, which were not considered, as natural spaces that intrinsically contributed benefits for the optimal development of cities, with the potential that its own characteristics offer, if not on the contrary, cataloged as inconvenient zones; remaining in the majority of cases abandoned, without attention from the authorities, turned into pockets of contamination and areas of insecurity for the cities, without carrying out a conscious attempt of the optimal integration of these, in the development plans of our cities in general. Thus, it is said, as mentioned above in Latin America, Europe, as well as in our Mexico, with several examples of urban infrastructure interventions for recreation and recreation, particularly linear urban parks, which as a new modality of public space They have been developed in different cities. Which are faithful examples of how it is intended, in an assertive way, to take advantage of and give a positive use to these natural areas, be they the river beds, ravines and bodies of water (lakes, dams, lakes, etc.).

\section{KEYWORDS}

Linear Park. Quality of Life. Livability. 


\section{Introducción}

En la planeación, diseño y materialización de los Parques Lineales, como de cualquier otro desarrollo urbano, presente en el crecimiento de nuestras ciudades, intervienen y deben de considerarse, las distintas variables que conforman el llamado mercado de tierras, de las cuales, las más significativas o de mayor injerencia, que podemos mencionar son las siguientes: la calidad de vida de los habitadores de los centros urbanos, las condiciones de habitabilidad y los cambios provocados en el suelo urbano, por su permuta de suelo urbano desocupado ha suelo urbano ocupado. Finalmente no pasar por alto el análisis de la relación de estos nuevos desarrollos urbanos con su contexto urbano inmediato, la manera en que estos influirán por sus características particulares, de qué manera intervendrán y modificaran el espacio urbano en el que se presentan.

En la planeación, diseño y materialización de los Parques Lineales, intervienen una gran cantidad de variables del llamado mercado de tierras, de las cuales las más significativas o de mayor injerencia, que podemos mencionar son: la calidad de vida de los habitadores, las condiciones de habitabilidad y el cambio del suelo urbano de desocupado ha ocupado; Así como, su posterior relación con el contexto en el que se encuentran, la manera en cómo influyen, intervienen y modifican el espacio urbano en el que se presentan.

\section{Precedentes urbanos internacionales}

De entre estos casos, comenzamos por mencionar los que se localizan en la ciudad de Medellín, Colombia, que se extienden a lo largo de la misma. Un artículo reciente (vid. infra) nos habla de cuatro de ellos: el parque la Presidenta, el parque La Bermejala, el parque La Hueso y el parque Bicentenario, y de cómo la población se ha apropiado de ellos. Dichos parques se desarrollan en las quebradas o cañadas y zonas contiguas que desembocan en el río Medellín, en el corazón de varios barrios de la ciudad, creados en el período de 2004 a 2007, con la finalidad de que funcionaran como elementos de transversalidad en el río, conectando los espacios oriental y occidental de la ciudad, generando espacios públicos, conservando la biodiversidad de los ecosistemas y logrando la descontaminación de las micro cuencas, pensando en la movilidad de los usuarios y peatones como actores principales, su recreación pasiva al aire libre, la apropiación individual y colectiva de éstos, además de llevar a cabo un rescate del medio ambiente donde se encuentran (Herrán 2012).

Otro de los ejemplos a nivel Latinoamérica es el del parque Gran Resistencia, ubicado en la provincia del Chaco, República Argentina; donde se llevó a cabo una apropiación de un ambiente natural con restricciones hídricas, que presentaba inundaciones periódicas de la región, en donde hubo necesidad de cambios para proteger a la población de las mismas y donde las políticas aplicadas permitieron transformar este espacio en un parque recreativo.

Ahí se llevaron a cabo obras físicas (4 plazas de $10,000 \mathrm{~m}^{2} \mathrm{c} / \mathrm{u}$, y una principal de 40,000 $\mathrm{m}^{2}$ ), medidas regulatorias del uso del suelo, y se recuperaron las lagunas existentes. Lo que creó un sistema de defensa contra las inundaciones y áreas verdes/recreativas, disponibles para la recreación de la población contigua al parque, creando un ambiente urbano-ambiental (Schneider 2010).

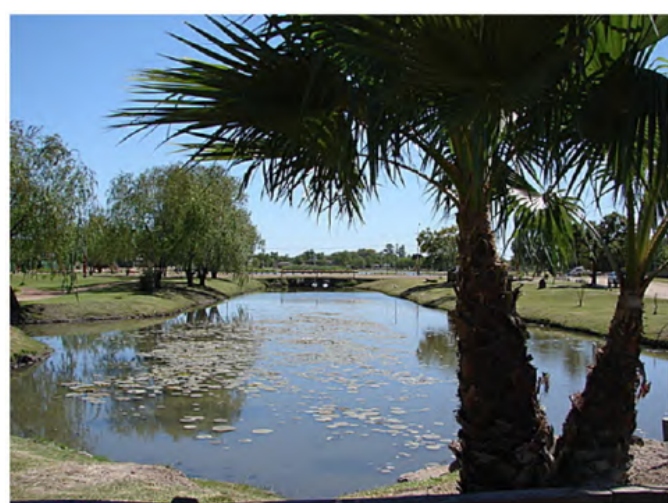

Figura 1. Parque Gran Resistencia. Fotografía: Mujica, 2010.

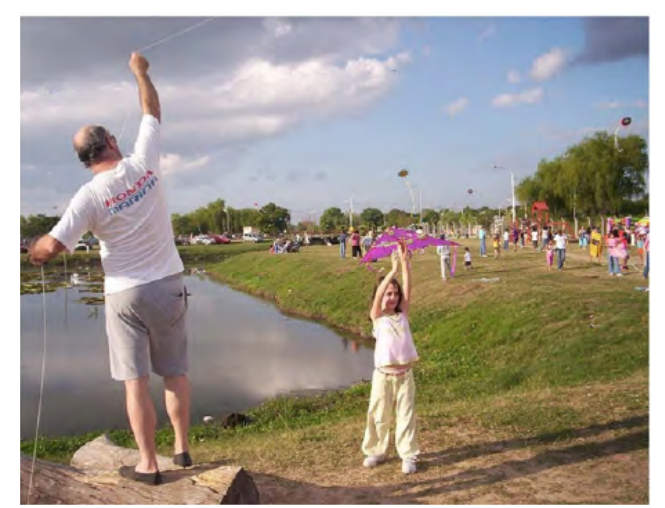

Figura 2. Parque Gran Resistencia. Fotografía: Flores, 2010.

También en América del Sur tenemos como ejemplo a la ciudad de Curitiba, en Brasil, ciudad que ha tomado como política pública la creación de parques lineales sobre las cuencas de los ríos y lagunas de contención paralelas a los ríos, que la circundan, el diseño de los cuales comenzó en la década de 1970 con la creación de su primer parque, el Barigui en 1972, y continuó con los parques, San Lorenzo y el Barreirinha en ese mismo año, además del parque Iguazú en el año de 1978. Estos parques, al mismo tiempo de proporcionar una 


\section{Labor \& Engenho}

alternativa de esparcimiento para la población, ayudan a controlar las inundaciones que padecía la ciudad; en ellos se han creado huertos públicos, un zoológico, un parque náutico, un parque de los jugadores de fútbol, el parque lineal Cajuru, la sede de la policía forestal, el parque centenario de la inmigración japonesa, además del museo de Historia Natural.

Dichos parques lineales de la ciudad de Curitiba están destinados a tener una doble función, la de uso urbano de esparcimiento y de recreación de la población (ya que cuentan con distintas áreas para realizar actividades tanto culturales, educativas, turísticas y deportivas), y la no menos importante de preservar el medio ambiente, logrando con ello mejorar en todos los aspectos la calidad de vida de sus pobladores (Fukuda 2010).

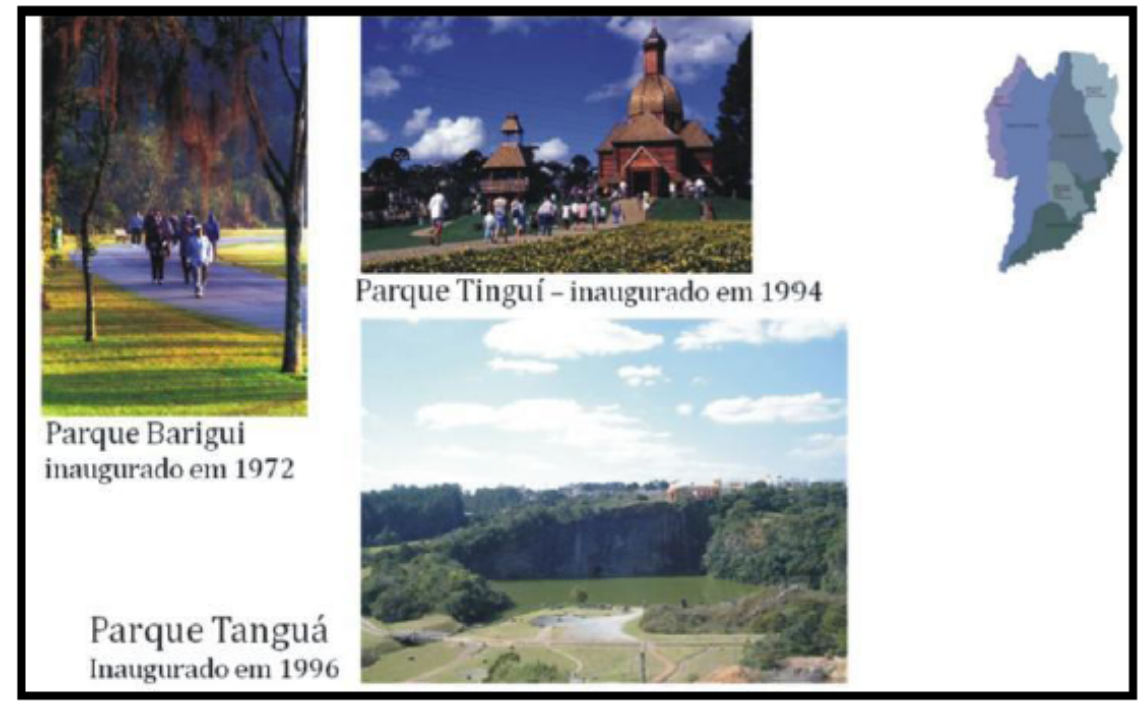

Figura 3. Parques ciudad de Curitiba Brasil. Fuente: IPPIUC, 2004.

En Argentina, en la ciudad de Puerto Madero, se encuentra un conjunto de parques que conforman un parque lineal, éstos son el Parque Micaela Bastidas, el parque Mujeres Argentinas y el parque María Eva Duarte de Perón. El primero de ellos busca reproducir con su aterrazamiento la línea de barranca que caracteriza a las principales plazas de la ciudad situadas en proximidad a la costa del río. Como parte del sistema de nuevas áreas verdes de Puerto Madero, el parque Micaela Bastidas cubre 7,2 has. Su geografía se define por movimientos de suelo que conforman desniveles que definen a partir de muros de gavión espacios de diferente escala y carácter, cuenta con tres plazas que se abren hacia la ciudad: la Plaza de los Niños, el Rosedal del Sur y la Plaza del Sol.

En él se plantaron 40.000 árboles, arbustos y herbáceas, casi todos de flor, y una mayoría de especies nativas que crean compactas masas de color.
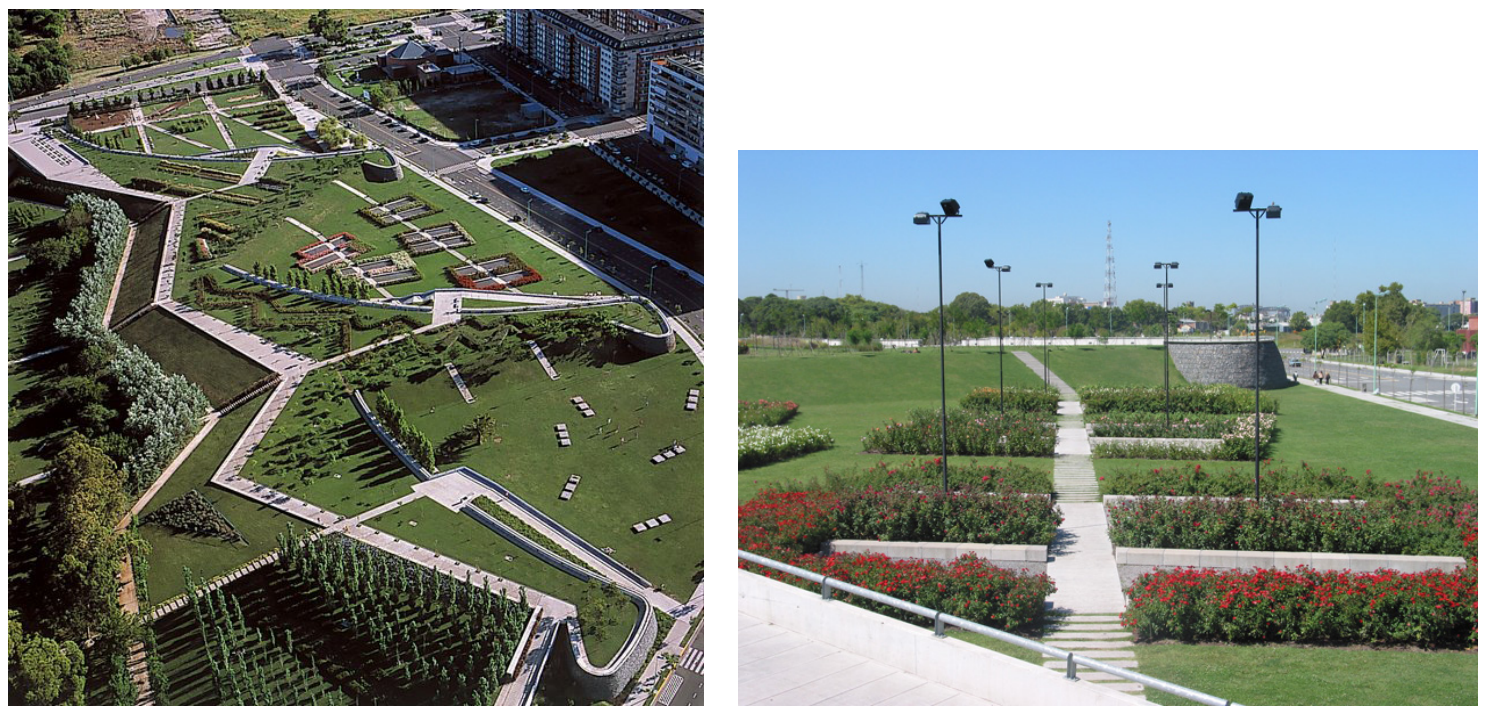

Figuras 4 y 5. Parque Micaela Bastidas. Fotografía: Puerto Madero Parques y Paseos, 2014. 


\section{Labor \& Engenho}
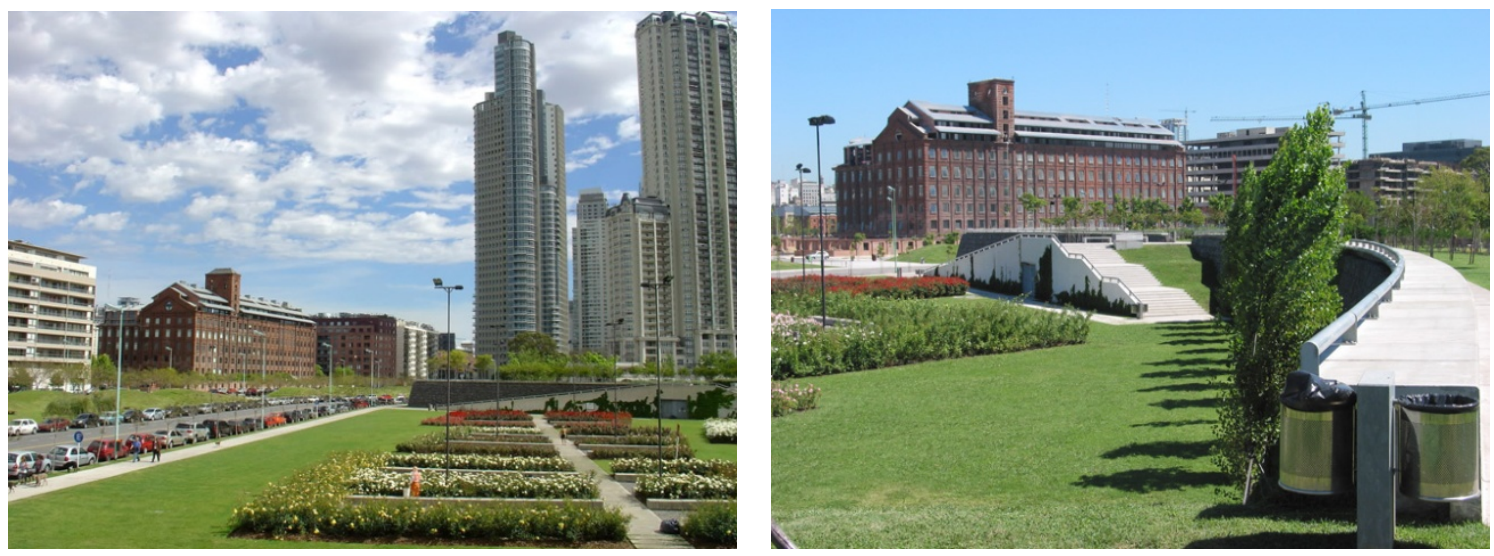

Figuras 6 y 7. Parque Micaela Bastidas. Fotografía: Puerto Madero Parques y Paseos, 2014.

El segundo parque, llamado Parque Mujeres Argentinas, se vincula y amarra la ciudad con el río, generando espacios de encuentro masivo y zonas de contención. Junto con el Micaela Bastidas y la plaza Raquel Forner conforman una unidad de proyecto, de operación sobre vacíos que, junto a la operación de recuperación del área histórica, estructura la nueva relación entre el borde natural y el construido.
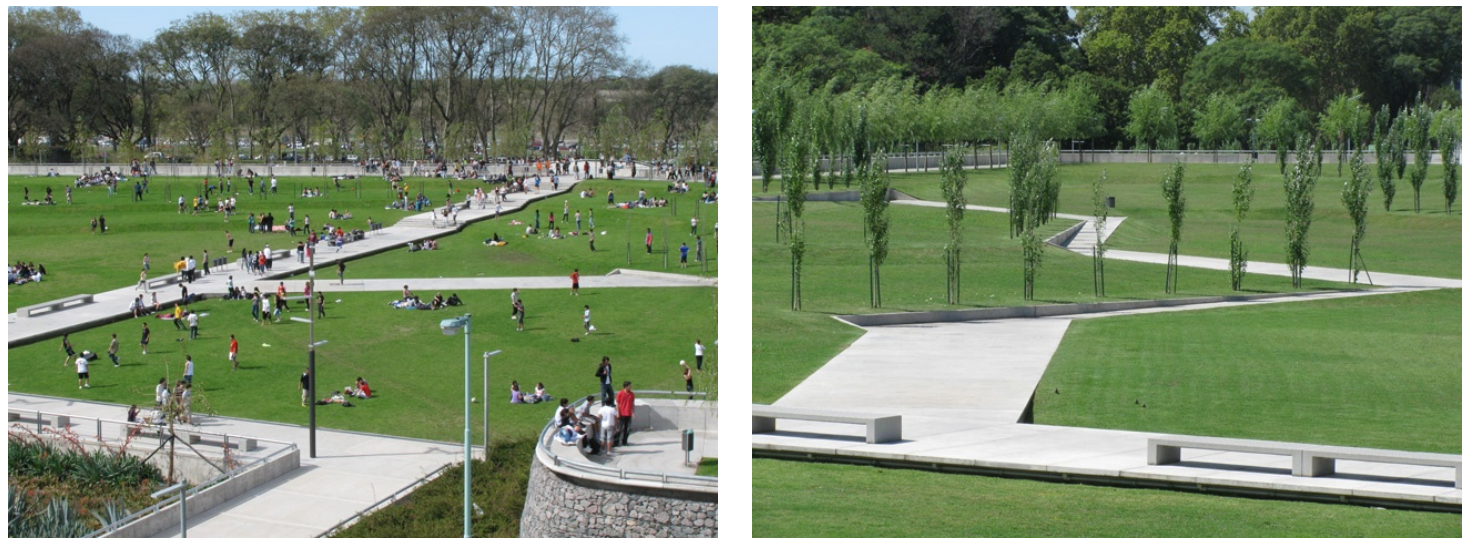

Figuras 8 y 9. Parque Mujeres Argentinas. Fotografía: Puerto Madero Parques y Paseos, 2014.

Por último, en este conjunto de parques de planteamiento lineal se encuentra el parque María Eva Duarte de Perón, que se extiende en una superficie de 4 hectáreas y que ayuda a consolidar el sistema de espacios verdes públicos de la ciudad de Puerto Madero y la Costanera Sur. El parque conforma dos grandes áreas estructuradas a partir del camino ondulante que atraviesa en forma longitudinal la manzana, formando patios con distintos programas: el Patio de los Rosales caracterizado por el color y el perfume de sus flores, y el Patio de Juegos infantiles donde se organizan equipos multijuego, la Plaza de los Frutales combina naranjos, limoneros, manzanos de flor, perales, nísperos y azahares, generando un área de descanso donde los sentidos son los protagonistas. Al sur se disponen tres plazas secas de adoquines recuperados del viejo puerto, con canteros de lagestroemias de distintos colores, flanqueadas por áreas verdes (Puerto Madero Parques y Paseos, 2014).
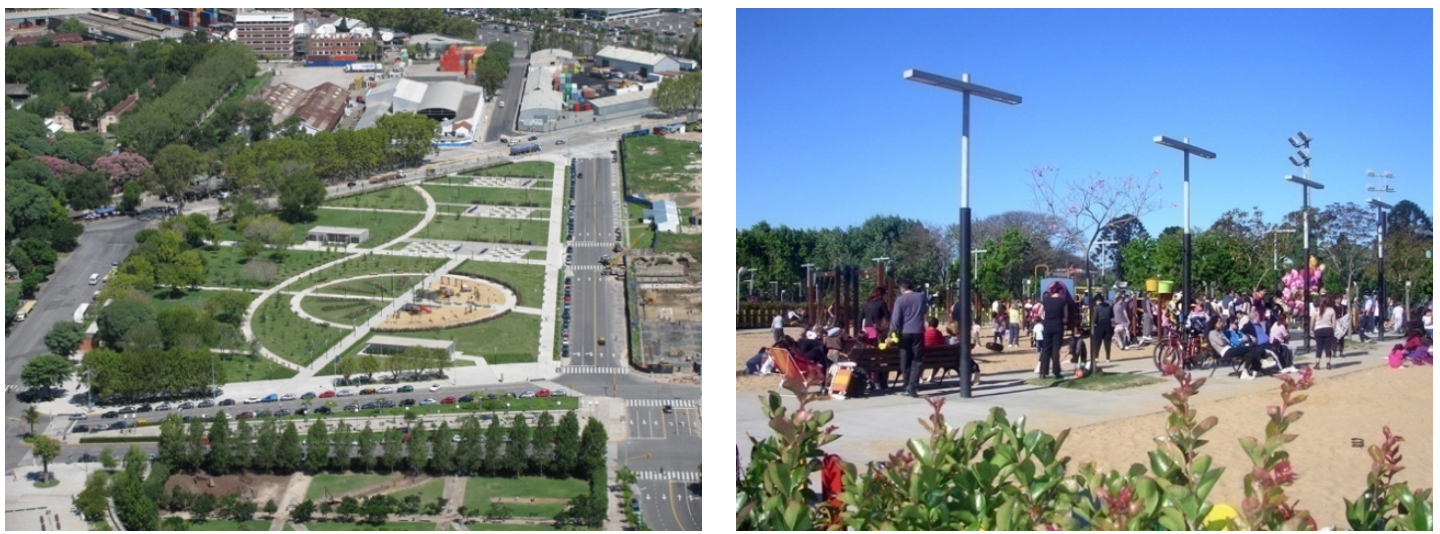

Figuras 10 y 11. Parque María Eva Duarte de Perón. Fotografía: Puerto Madero Parques y Paseos, 2014. 


\section{Labor \& Engenho}
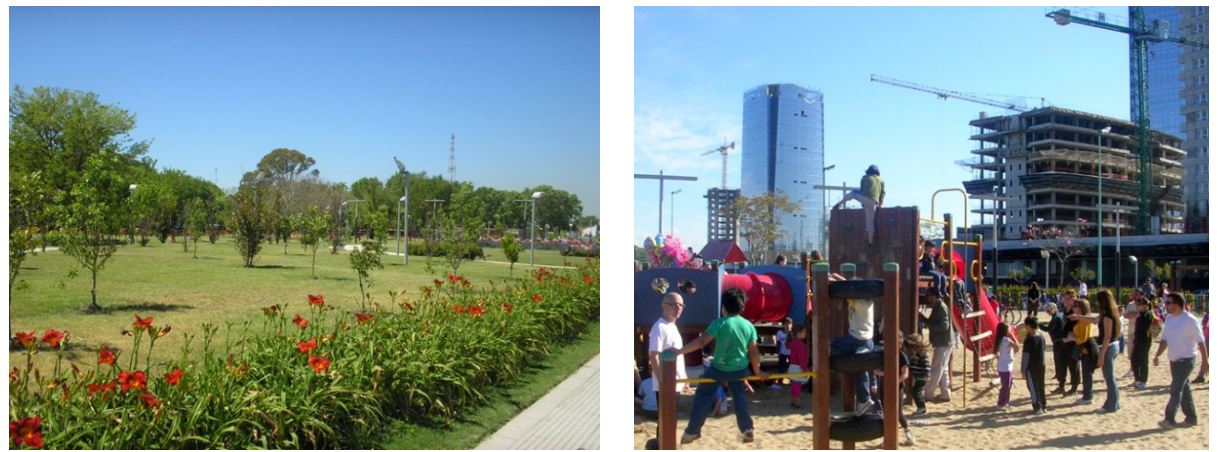

Figuras 12 y 13. Parque María Eva Duarte de Perón. Fotografía: Puerto Madero Parques y Paseos, 2014.

De Europa podemos citar el ejemplo del Parque Lineal Del Manzanares, en Madrid, España, el cual surge del soterramiento de parte del anillo de la M-30, una de las carreteras que circunvalan la capital de España y la recuperación del cauce del río Manzanares en su paso por el centro de la ciudad; abarca un poco más de 80 hectáreas, que dotaron a la ciudad de espacios deportivos (circuitos biosaludables, pistas de tenis y pádel, skatepark y patinaje, etc.), áreas de juegos de niños, una playa urbana e itinerarios ciclistas que acompañan al llamado Salón de Pinos, un paseo arbolado de $6 \mathrm{~km}$ de longitud. El parque, además de crear nuevas pasarelas y espectaculares puentes, como los Gemelos o el Monumental de la Arganzuela, integra los ya existentes, como los históricos de Segovia y Toledo; su periodo de construcción abarcó de 2005 a 2011 (Dtlux.com Dolce Vita, 2014).

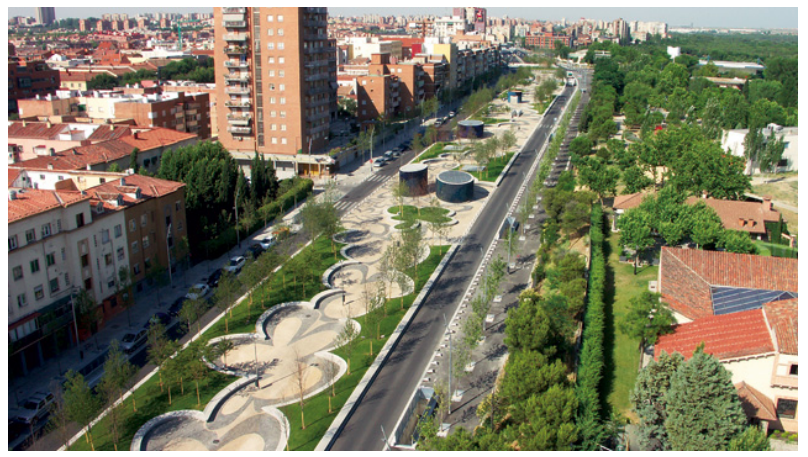

Figura 14. Parque Lineal Del Manzanares. Fotografía: Dtlux.com Dolce Vita, 2014.
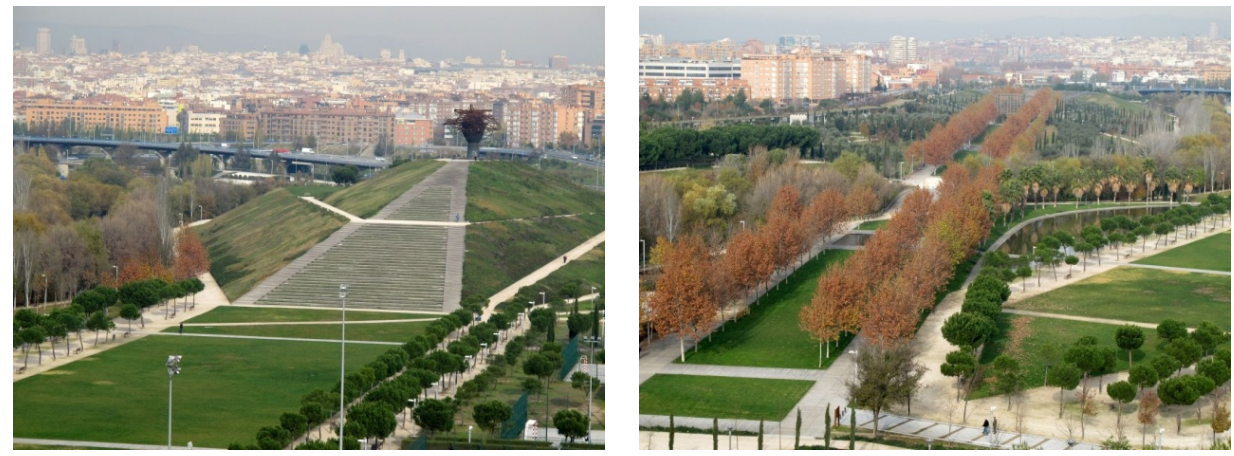

Figuras 15 y 16. Parque Lineal Del Manzanares. Fotografía: (Dtlux.com Dolce Vita, 2014).
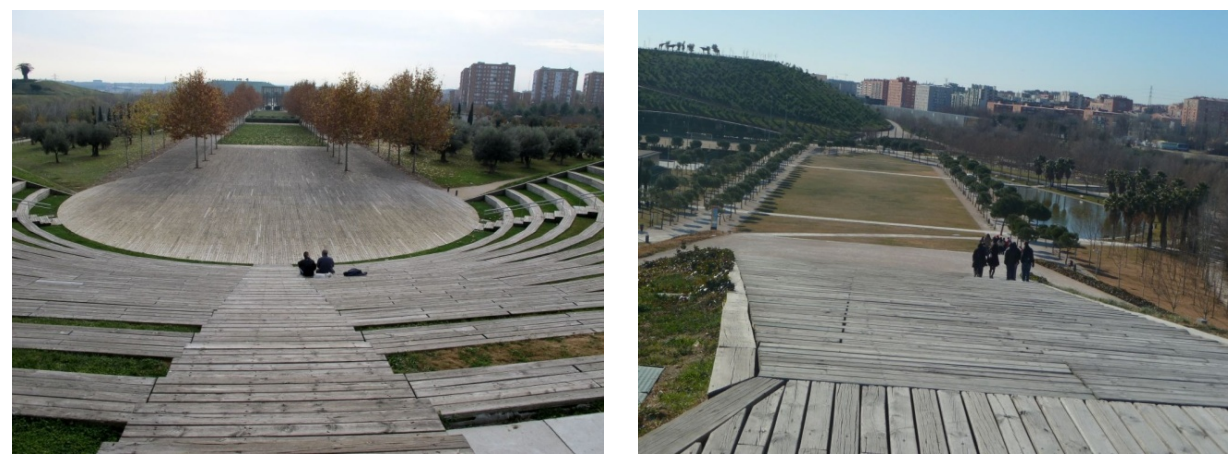

Figuras 17 y 18. Parque Lineal Del Manzanares. Fotografía: Dtlux.com Dolce Vita, 2014. 
Otro de los ejemplos que podemos citar de Europa es el del Parque Lineal Tagus, localizado en la ciudad de Póvoa de Santa Iria, Portugal, el cual se desarrolla en un área de $15000 \mathrm{~m} 2$ y cuyo proyecto y construcción son del año 2013. El Parque Lineal Tagus es un área que fue ganada por las comunidades del entorno al sector privado industrial, lo que se sintió como una intervención democrática para aquellos que habían estado siempre privados del acceso al río. Por primera vez, la gente de las comunidades urbanas adyacentes tuvo acceso a la recreación y la oportunidad de contar con un espacio en contacto directo con la orilla del río, que estaba hasta hace muy poco tiempo bloqueada por grandes lotes industriales. Personas de todas las edades, de diferentes estilos de vida y culturas están invitados a disfrutar de una diversa variedad de equipamientos y actividades: desde deportes, pesca, senderismo y ciclismo, educación ambiental, o simplemente para observar un hermoso paisaje.

El parque combina dos tipologías diferentes de espacios: un área multifuncional llamada "Praia dos Pescadores" (Playa de los Pescadores), situada en la orilla del río y 6 kilómetros de caminos peatonales, bancos de líneas de agua (arroyos y zanjas de drenaje), que convergen en la "Praia dos Pescadores", provenientes de áreas urbanas y naturales. La conexión entre la "playa" y las áreas naturales se logra a través de un sendero aéreo de madera de $700 \mathrm{~m}$ de largo, desde el cual se puede llegar a un observatorio de aves. La "Praia dos Pescadores" contiene equipamientos diversos y complementarios destinados principalmente a la educación ambiental, el ocio y deportes informales, como las plataformas de pesca y refugios, zonas de picnic, una cancha de voleibol, un sencillo patio de juegos hecho de neumáticos reciclados, así como plataformas para tomar el sol, en sus 3 hectáreas de frente al río, generando un interesante y único paisaje lúdico. La iluminación del parque es 100\% solar (Archdaily Parque Linear Tagus / Topiaris Landscape Architecture, 2014).

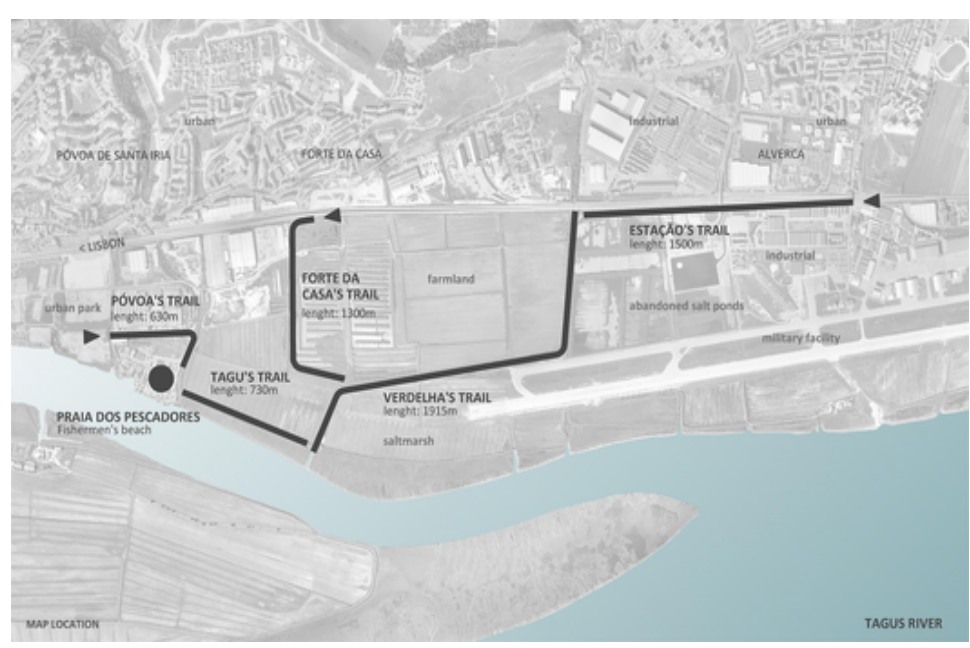

Figura 19. Parque Lineal Tagus, Póvoa de Santa Iria, Portugal. Fuente: Archdaily Parque Linear Tagus / Topiaris Landscape Architecture, 2014.
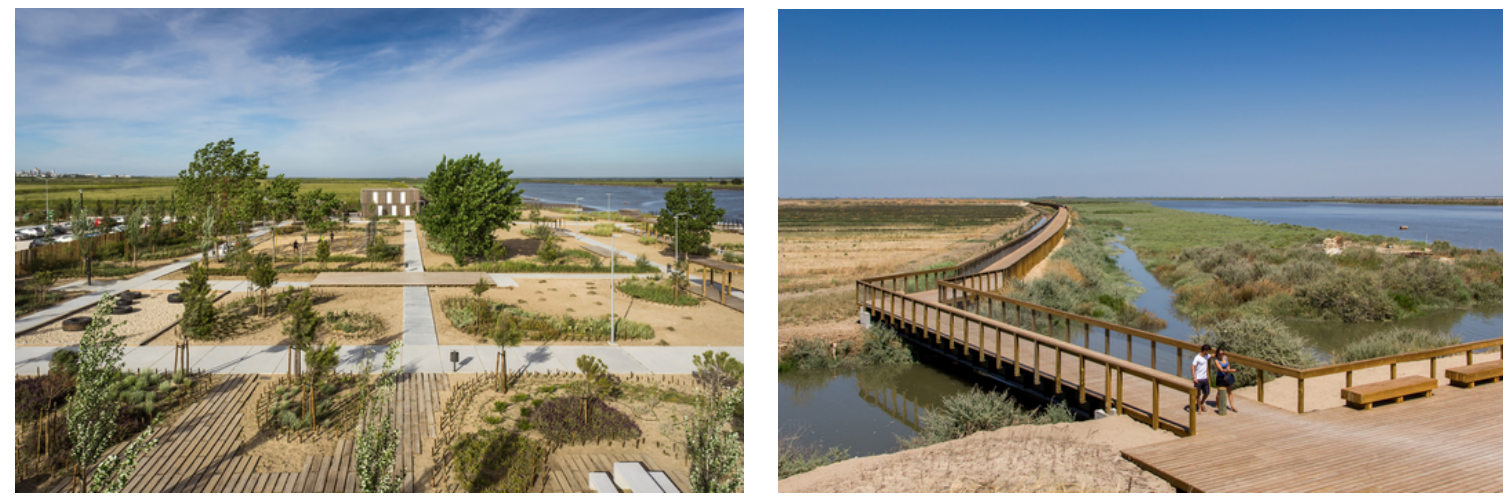

Figuras 20 y 21. Parque Lineal Tagus, Póvoa de Santa Iria, Portugal. Fotografía: Archdaily Parque Linear Tagus / Topiaris Landscape Architecture, 2014.

\section{Precedentes urbanos nacionales}

A nivel nacional podemos mencionar el de la ciudad de Puebla, la cual cuenta con su "Parque lineal", el cual tiene como atractivo principal una rueda de la fortuna llamada Rueda de Observación y una fuente adyacente a la misma llamada La Fuente Estrella de la ciudad de Puebla; el parque se localiza en terrenos propios del 
estado, en él se puede disfrutar de espacios ecológicos y de convivencia, contando con una vista panorámica de la ciudad, los volcanes y la zona de Cholula.

Este proyecto integral incluye la recuperación ecológica de áreas verdes que conectan las instalaciones del Parque del Arte con las del Centro Integral de Servicios (CIS), en una superficie de dos hectáreas, contando con corredores y andadores peatonales, así como pistas de trote y ciclovías con una longitud de 1.5 kilómetros. Desarrollado en 7.5 hectáreas, en las cuales se utilizan pavimentos ecológicos y materiales pétreos, permitiendo una mayor absorción del agua y el cuidado y preservación del medio ambiente. Contando con un estacionamiento con capacidad para 1200 vehículos (Parque Lineal Puebla, 2014).

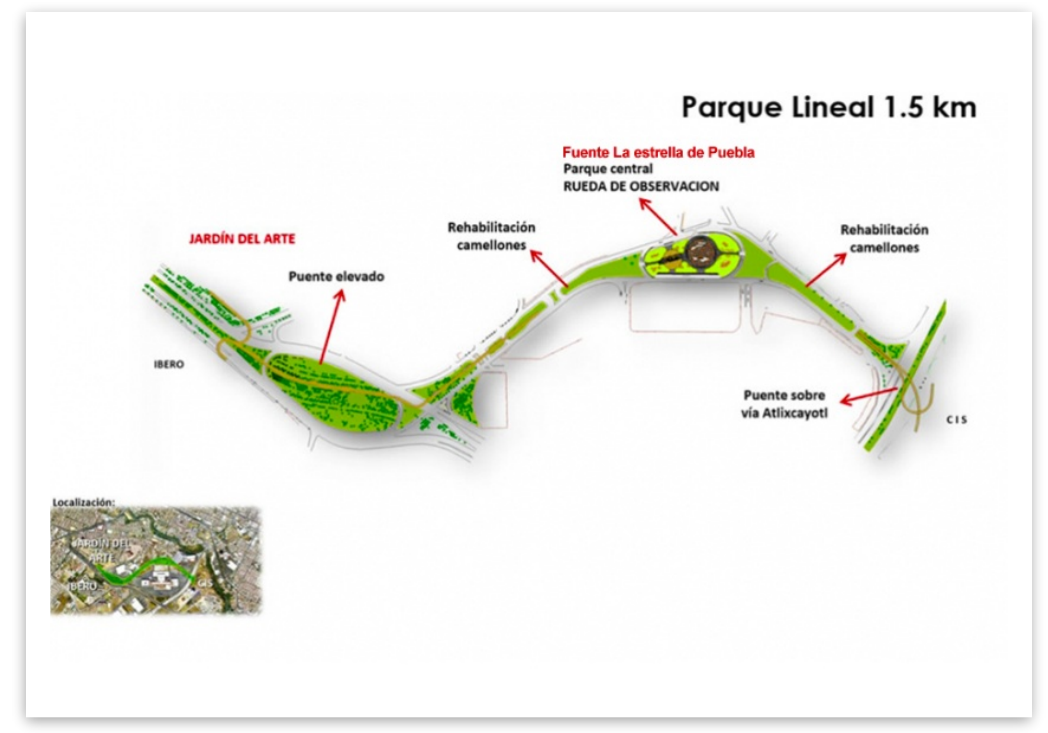

Figura 22. Parque Lineal Puebla, México. Fuente: Parque lineal puebla, 2014.

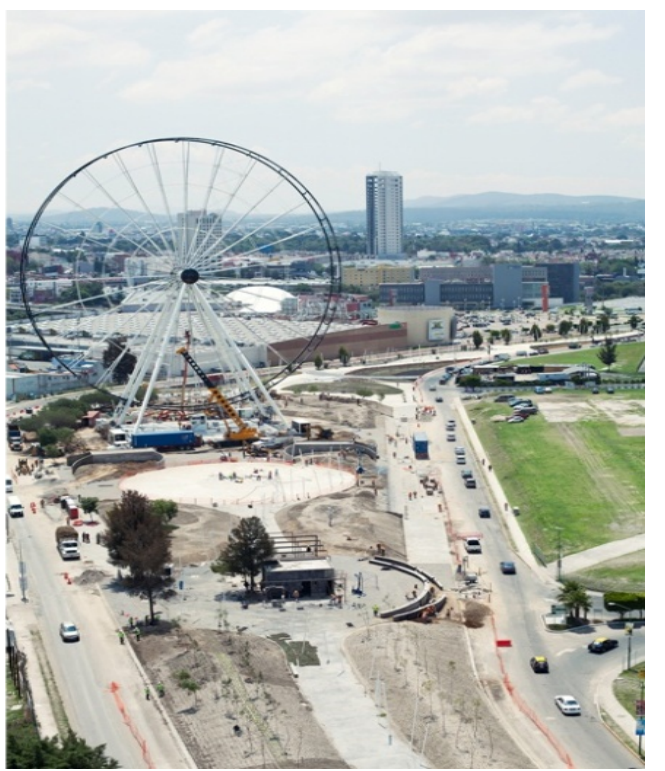

Figura 23. Parque Lineal Puebla, México. Fotografía: Parque Lineal Puebla, 2014.

Otro de los ejemplos a nivel nacional es el Parque lineal del Río Santa Catarina, en la ciudad de Monterrey, Nuevo León, que se extiende en una primera etapa del puente Gonzalitos hasta el puente Revolución en una longitud aproximada a los 9 kilómetros, el cual se planea a futuro como un macroparque de 45 kilómetros de largo, abarcando desde La Huasteca hasta el municipio de Juárez, en 770 hectáreas, con una inversión aproximada de mil 100 millones de pesos, el cual contará con áreas verdes, una playa artificial, instalaciones deportivas, reservas ecológicas, espacios recreativos y un espejo de agua de un metro de profundidad en todo el recorrido del río (Monterrey, México Parque Lineal Río Santa Catarina formalmente inaugurado 2007).

Además de contar con 12 mil cajones de estacionamiento, 30 áreas deportivas, seis zonas de juegos infantiles, tres espacios culturales, tres puentes peatonales, un vivero y la rehabilitación del Mercado San Luisito. 


\section{Labor \& Engenho}

Pretendiendo aumentar el promedio de áreas verdes en la zona metropolitana de 4 a 7 metros cuadrados por habitante. Por lo pronto esta obra cuenta con una ciclopista y trotapista, paisajismo con plantas y árboles nativos de la región, así como con espacios deportivos y recreativos (Skyscrapercity.com zmm / Macroparque Río Santa Catarina, 2006).
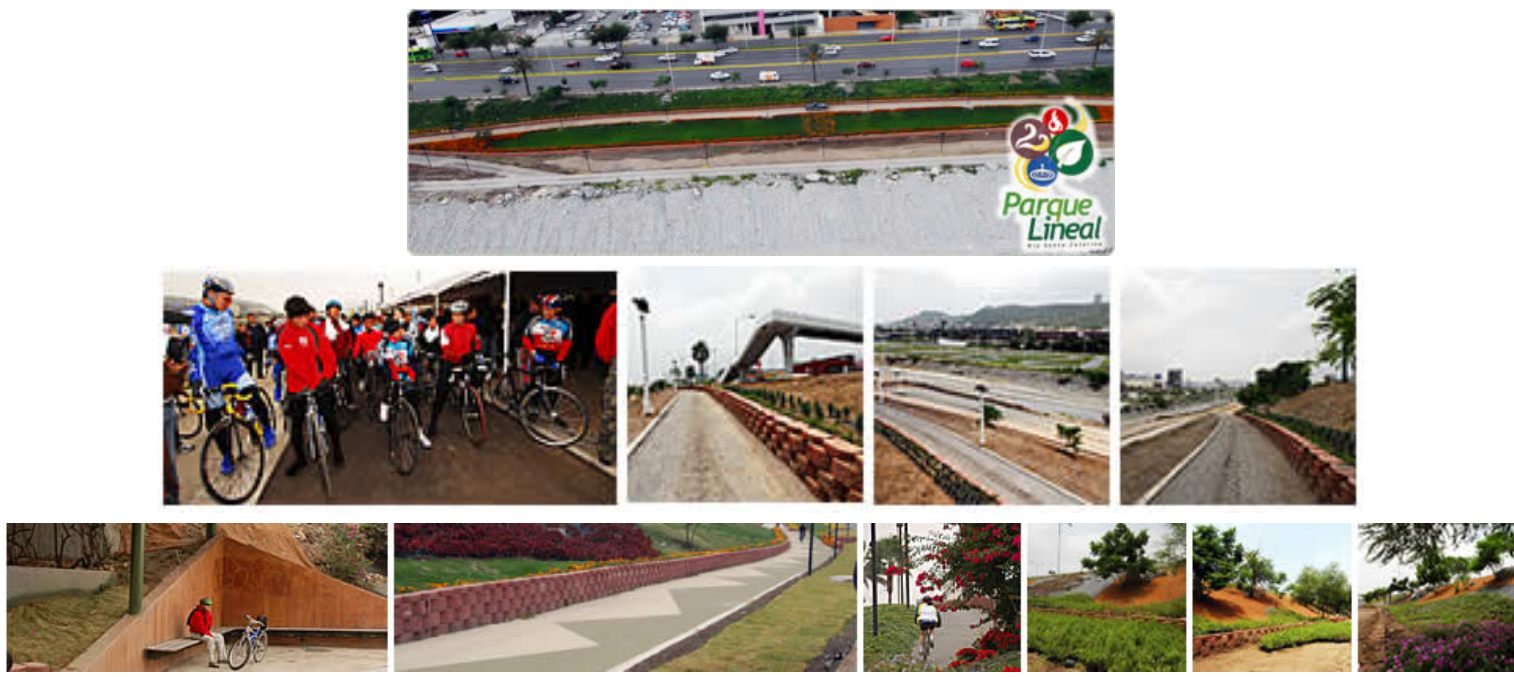

Figura 24. Parque Lineal Río Santa Catarina, Monterrey, Nuevo León, México. Fuente: Nuevo León Unido / Gobierno Para Todos,

Parque Lineal del Río Santa Catarina, 2014.
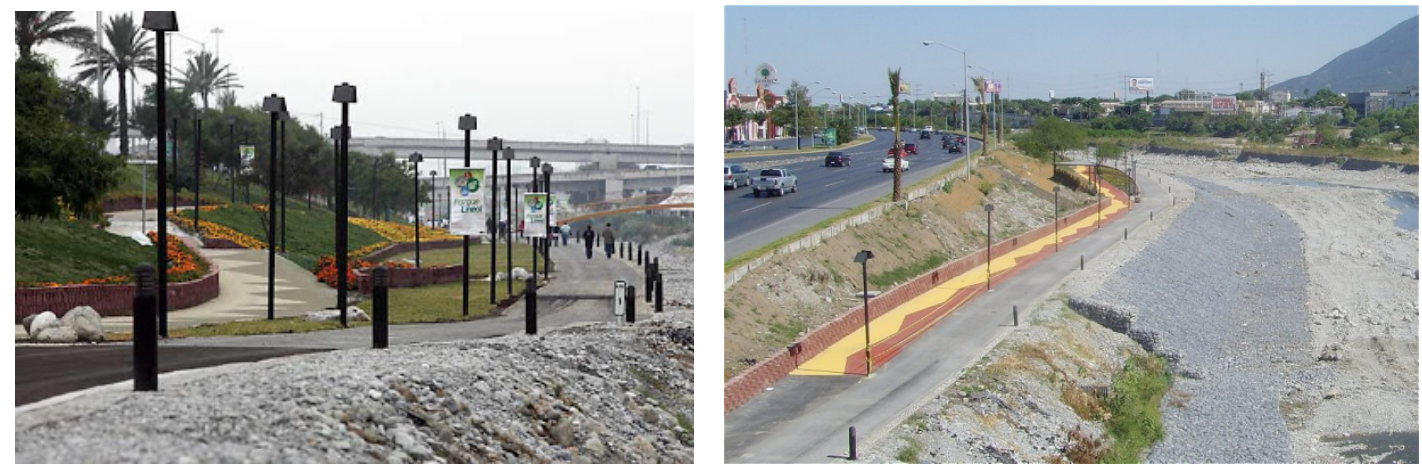

Figuras 25 y 26. Parque Lineal Río Santa Catarina, Monterrey, Nuevo León, México. Fotografía: skyscrapercity.com ZMM / Macroparque Río Santa Catarina, 2006.

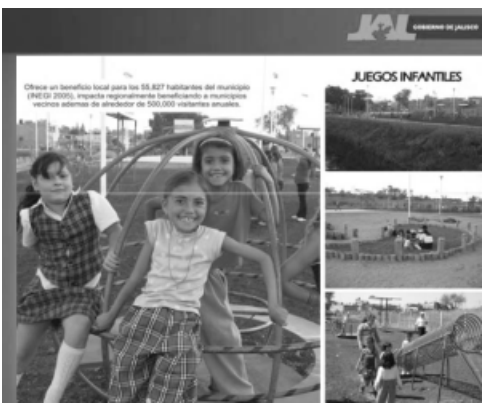

El Parque lineal de Zapotlanejo, Jalisco, es otro de los ejemplos a mencionar, ubicado aproximadamente a 40 minutos de la Zona Metropolitana de Guadalajara, cuya construcción se realiza entre los años 2007 al 2009.

Figura 27. Parque Lineal Zapotlanejo, Jalisco,

México. Fuente: Tiempos de Enfoque. Otro

Espacio Público: El Parque Lineal, 2010.
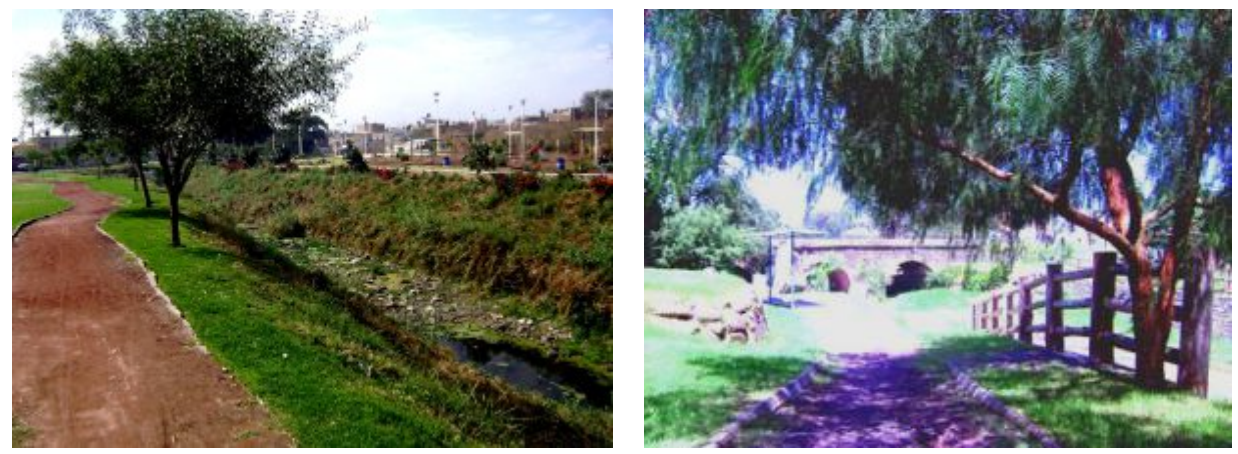

Figuras 28 y 29. Parque Lineal Zapotlanejo, Jalisco, México. Fotografía: Tiempos de Enfoque, Otro Espacio Público: El Parque Lineal, 2010. 


\section{Labor \& Engenho}

La infraestructura se compone de 22 kilómetros de áreas verdes, andadores, fuentes, juegos infantiles, canchas de básquetbol y voleibol, pista de skate, aparatos de ejercicio, una ciclovía, un corredor escultórico y un auditorio al aire libre con capacidad para mil quinientas personas; el parque se desarrolla a lo largo del Río "La Peñita" y "Zapotlanejo". El andador del parque comienza en la colonia San Martín hasta llegar a la colonia San Francisco; el recorrido lineal lleva a dos destinos: El Parque Escultórico y el Camino Real al Parque del Bicentenario Puente de Calderón (Tiempos de Enfoque Otro Espacio Público: El Parque Lineal, 2010).
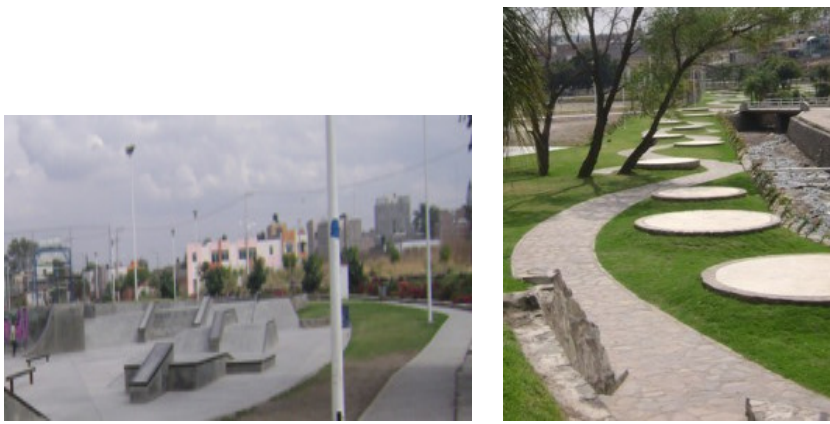

Figuras 30 y 31. Parque Lineal Zapotlanejo, Jalisco, México. Fotografía: Tiempos de Enfoque Otro Espacio Público: El Parque Lineal, 2010.

Finalmente podemos mencionar al Parque lineal, Río La Laja ubicado en Celaya, Guanajuato, abierto al público en su primera etapa a partir de julio del 2009, el cual comienza en el puente Tres Guerras, sobre el Río La Laja, con una extensión en su primera etapa de $2.2 \mathrm{~km}$, cuyo proyecto comprende una extensión de $8.4 \mathrm{~km}$ (Celaya y sus fotos Parque Lineal Celaya 2011).
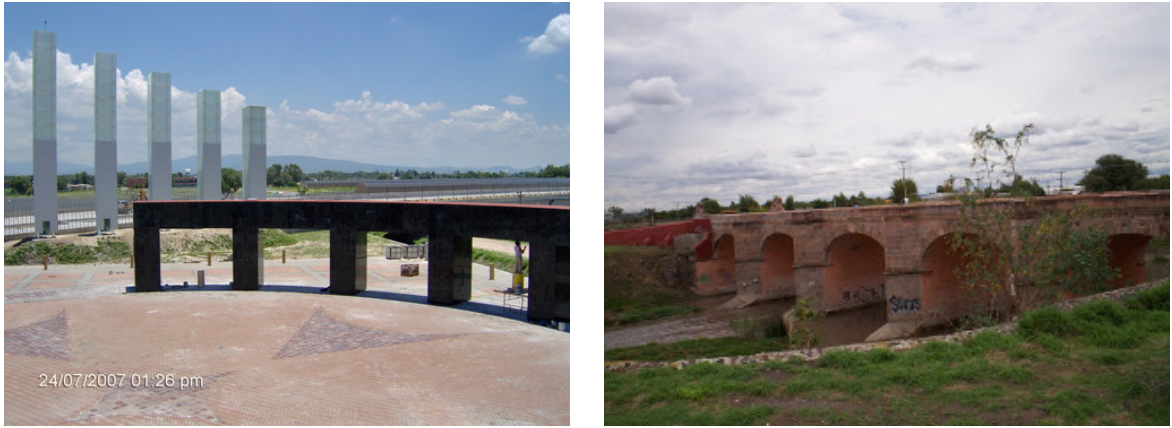

Figuras 32 y 33. Parque Lineal, Río La Laja. Fotografía: geoview.info Parque Lineal Río Laja, Macroplaza de Acceso, 2007.
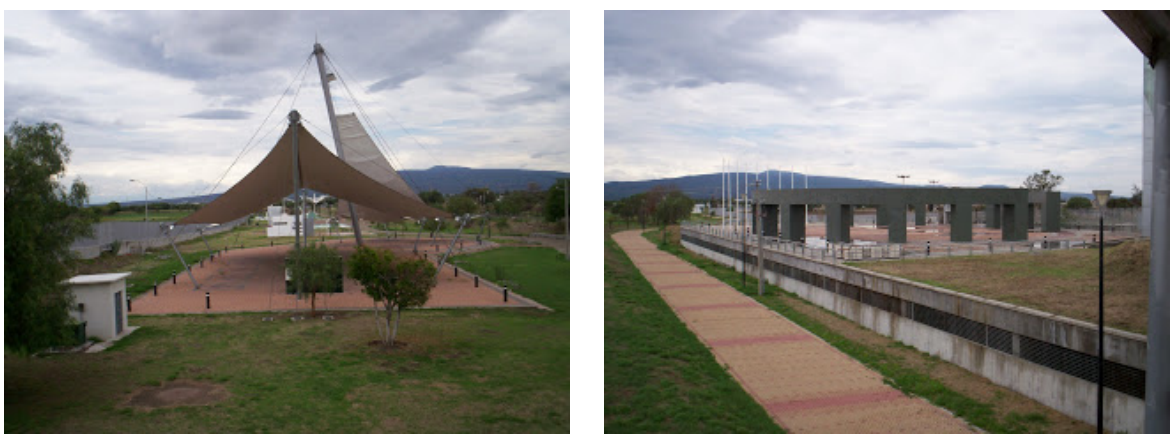

Figuras 34 y 35. Parque Lineal, Río La Laja. Fotografía: geoview.info Parque Lineal Río Laja, Macroplaza de Acceso, 2007.
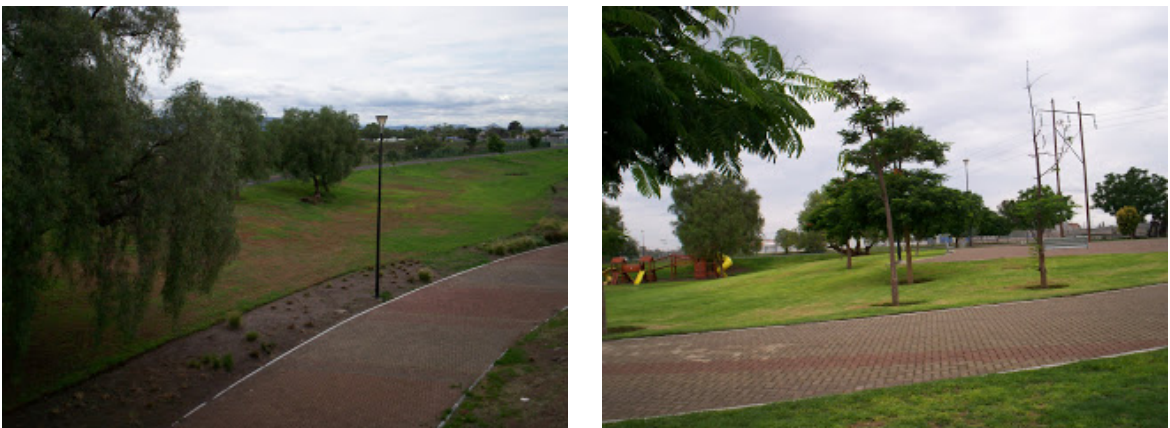

Figuras 36 y 37. Parque Lineal, Río La Laja. Fotografía: Geoview.info Parque Lineal Río Laja, Macroplaza de Acceso, 2007. 


\section{Conclusiones}

Sin pretensión de exhaustividad, éstas son algunas de las experiencias de parques lineales que se han llevado a cabo en distintas partes del mundo. En todas ellas se destaca, que más allá del equipamiento con el que se dotan estos parques, existe una persistente presencia de algunas variables que sus promotores y realizadores debieron tomar en cuenta en su planificación, la más importante de las cuales, en esencia, se relaciona con aquel elemento sin el cual no sería posible su existencia: el suelo, trátese éste del urbano o bien del rural, además de otras variables conexas que a él atañen, como lo son, la propiedad, el mercado, la rentabilidad, la especulación, las políticas urbanas y territoriales y las propias del ambiente en términos de sostenibilidad, que deben ser analizadas e incluidas en el diseño y elaboración de estos proyectos y que de haber sido incluidas y valoradas de manera más aguda en su realización, habría dado como resultado un mayor y mejor éxito en los resultados y preservación de estos parques.

\section{Bibliografía}

Archdaily Parque Linear Tagus / Topiaris Landscape Architecture (2014). Recuperado de: http://www.archdaily.mx/ $\underline{\mathrm{mx} / 02-370880 / \text { parque-linear-tagus-topiaris-landscape-architecture. }}$

Ardila, R. (2003). Calidad de vida: una definición integradora, en Revista Latinoamericana de Psicología, 35(2), Fundación Universidad Konrad Lorenz, Colombia, pp. 161-164.

Castells, M. (1976). La cuestión urbana [trad. I. C. Oliván; rev. de M. Castells]. México [D.F.]: Siglo Veintiuno Editores.

Castro Ramírez, M. E., Romero Castillo, L. I., Borre Aguilera, C. A., \& Anguiano Reyes, C. A. (2001). Habitabilidad, medio ambiente y ciudad, Revista Ciudades, 51, julio-septiembre, Puebla, México: RNIU.

Celaya y sus Fotos. Parque Lineal Celaya 2011 (2011). Recuperado de: http://celayaysusfotos.blogspot.com/ 2011/07/parque-lineal-celaya.html.

Discoli, C., San Juan, G., Martini, I., Ferreyro, C., Dicroce, L., Barbero, D., \& Esparza, J. (2010). Metodología para la evaluación de la calidad de vida urbana, en Revista Bitácora Urbano Territorial, 17(2), Colombia: Universidad Nacional de Colombia, julio-diciembre, pp. 95-112.

Dtlux.com Dolce Vita (2014). Recuperado de: http://www.dtlux.com/dolcevita/arteyarquitectura/articulo/parquesurbanos.

Fausto, A., \& Rábago, J. (2001). ¿Vacíos urbanos o vacíos de poder metropolitano?, en Revista Ciudades, 49, eneromarzo, Puebla, México: RNIU.

Fariña Tojo, J. (2006). Formas de regulación de la escena urbana en varias ciudades Europeas, en Cuadernos de investigación urbanística, $\mathrm{n}^{\circ}$ 48, Madrid: Departamento de Urbanística y Ordenación del Territorio, Universidad Politécnica de Madrid.

Fukuda Hayakawa, L. (2019). Planeación Urbana en Curitiba, en Quivera, 12(1), enero-junio, México: Universidad Autónoma del Estado de México, pp. 52-69.

Geoview.info Parque Lineal Río Laja - Macroplaza de Acceso (2007). Recuperado de: http://mx.geoview.info/ parque_lineal_rio_laja_macroplaza_de_acceso,3862676p

Goycoolea Infante, R. (2004). Ciudad versus calidad de vida, en Urbano, 7(9), Chile: Universidad del Bío Bío, mayo, pp. 18-25.

Hernández Aja, A. (2009). Calidad de vida y medio ambiente urbano. Indicadores locales de sostenibilidad y calidad de vida urbana, en Revista INVI, 24(65), Chile: Universidad de Chile, mayo, pp. 79-111.

Herrán, C. (2012). Los parques lineales como una nueva modalidad de espacio público inclusivo en la ciudad de Medellín, en Revista S\&T, 10(22), Memorias: 5to. Encuentro Internacional de Investigación en Diseño - Diseño + 2012, pp. 159-166.

López Rangel, R. (1982). Planificación para los monopolios o planificación para el pueblo, México: UAM/Xochimilco.

López Rangel, R. (1975). Arquitectura y subdesarrollo en América Latina (primer acercamiento al problema), Puebla, Pue.: Departamento de Investigaciones Arquitectónicas y Urbanísticas del Instituto de Ciencias de la Universidad Autónoma de Puebla. 
López Rangel, R., \& Segre, R. (1986). Tendencias arquitectónicas y caos urbano en Latinoamérica, México: Ediciones G. Gili.

Méndez Sáinz, Eloy (Coord.). 2007. Arquitecturas de la globalización, España: Universidad de Sonora.

Monterrey, México Parque Lineal Río Santa Catarina formalmente inaugurado (2007, Noviembre). Recuperado de: http:// fermintellez.blogspot.com/2007/11/parque-ro-formalmente-inaugurado.html.

Moreno Olmos, Silvia Haydeé (2008). La Habitabilidad urbana como condición de calidad de vida, en Palapa, III, No. II, México: Universidad de Colima, julio-diciembre, pp. 47-54.

Nuevo León Unido / Gobierno para todos. Parque Lineal del Río Santa Catarina (2014). Recuperado de: http:// www.nl.gob.mx/?P=forum_parquelineal.

Parque Lineal Puebla (2014). Recuperado de: http://www.parquelineal.com/.

Pérez Maldonado, A. (1999). La construcción de indicadores bioecológicos para medir la calidad del ambiente natural urbano. Mérida [Venezuela]: Facultad de Arquitectura y Arte de la Universidad de los Andes.

Puente, S., \& Legorreta, J. (Coords.) (1988). Medio ambiente y calidad de vida, México: Plaza y Valdés.

Puerto Madero, Parques y Paseos (2014). Recuperado de: http://www.puertomadero.com/parques esp.php.

Rodríguez Salgado, M. C. (2002). Manejo de áreas verdes en Concepción: mejor calidad de vida urbana. Urbano, 5(6), agosto, 2002, pp. 41-42, Universidad del Bío Bío, Chile.

Rojas, M., Méndez, R., \& Rodríguez, Á. (2012). Subjetividad en la evaluación de la calidad de vida en contextos urbanos. Revista Virtual Universidad Católica del Norte, 37, septiembre-diciembre, 2012, pp. 212-235. Fundación Universidad Católica del Norte, Medellín, Colombia.

Rozas, G. (2002). Efectos psicosociales, ciudad y calidad de vida. Psychosocial Intervention, 11(2), 2002, pp. 229-243, Colegio Oficial de Psicólogos de Madrid, España.

Schneider, V. (2010). Cambios cualitativos en infraestructuras urbanas. Recuperación de ambientes naturales degradados en el área metropolitana del Gran Resistencia, Argentina. Revista Bitácora Urbano Territorial, 17(2), juliodiciembre, 2010, pp. 113-126. Universidad Nacional de Colombia. Bogotá, Colombia.

Skyscrapercity.com Zmm / "Macroparque Río Santa Catarina” (2006, Abril). Recuperado de: http:// www.skyscrapercity.com/showthread.php?t=335055.

Tiempos de Enfoque. “Otro Espacio Público: El Parque Lineal” (2010, Julio). Recuperado de: http:// tiemposdeenfoque.wordpress.com/2010/07/19/otro-espacio-publico-el-parque-lineal/

Timmling, H. F. (2008). Un orden urbano: paisaje, calidad de vida y sustentabilidad. Urbano, 11(17), mayo, 2008, pp. 89-97, Universidad del Bío Bío, Chile.

Vila Planes, E. (1998). Diseñar-planificar: Fundamentos e Ideas. Tesis de Doctorado en Arquitectura y Urbanismo, Mención Diseño, Facultad de Arquitectura y Urbanismo, Universidad Central de Venezuela, Caracas.

Ziccardi, A. (Coord.) (1991). Ciudades y gobiernos locales en la América Latina de los noventa. México: Instituto Mora: Miguel Ángel Porrúa: Facultad Latinoamericana de Ciencias Sociales.

Zulaica, L., \& Celemín, J. P. (2008). Análisis territorial de las condiciones de habitabilidad en el periurbano de la ciudad de Mar del Plata (Argentina), a partir de la construcción de un índice y de la aplicación de métodos de asociación espacial. Revista de Geografía Norte Grande, 41, pp 129-146. Universidad Nacional de Mar del Plata, CONICET (Argentina). 